\title{
Micropolar Fluid Flow and Heat Transfer over a Nonlinearly Stretching Plate with Viscous Dissipation
}

\author{
Kartini Ahmad, ${ }^{1}$ Anuar Ishak, ${ }^{2}$ and Roslinda Nazar ${ }^{2}$ \\ ${ }^{1}$ Department of Science in Engineering, Kulliyyah of Engineering, International Islamic University Malaysia, Gombak, \\ 50728 Kuala Lumpur, Malaysia \\ ${ }^{2}$ School of Mathematical Sciences, Faculty of Science and Technology, Universiti Kebangsaan Malaysia, 43600 Bangi, \\ Selangor, Malaysia \\ Correspondence should be addressed to Anuar Ishak; anuarishak@yahoo.com
}

Received 1 March 2013; Accepted 12 June 2013

Academic Editor: Xing-Gang Yan

Copyright ( $\odot 2013$ Kartini Ahmad et al. This is an open access article distributed under the Creative Commons Attribution License, which permits unrestricted use, distribution, and reproduction in any medium, provided the original work is properly cited.

\begin{abstract}
The flow and heat transfer of a micropolar fluid past a nonlinearly stretching plate is studied numerically, by taking into account the viscous dissipation effect. It is assumed that the plate is stretched nonlinearly from the slot where it is issued. The governing system of partial differential equations is transformed into ordinary differential equations, which are then solved numerically using a finite-difference scheme known as the Keller-box method. The effects of the governing parameters, namely, the material parameter $K$, the Eckert number Ec, the Prandtl number Pr, and the nonlinear stretching parameter $n$, on the flow field and the heat transfer characteristics are obtained and discussed. The velocity and the temperature profiles are also illustrated to aid the validity of the numerical results obtained. It is found that both the local Nusselt number and the magnitude of the skin friction coefficient increase with the nonlinear stretching parameter $n$, and the opposite trend occurs as $K$ increases for fixed $n$.
\end{abstract}

\section{Introduction}

The initial study by Crane [1] has attracted many researchers to investigate similar problems on the boundary layer flow caused by a stretching sheet, as it has a number of applications in industry such as the extrusion of polymer sheet from a dye, continuous casting, drawing of plastic films, and crystal growing. The desired characteristics of the final product strictly depend on the rate of cooling and the process of stretching. This stretching sheet may not necessarily be linear, as it can also take nonlinear fashion, even though the problem might not have obvious technological relevance [2]. In view of this, Vajravelu [3] studied the flow over a nonlinearly stretching sheet, and Cortell $[4,5]$ examined the flow and heat transfer on a nonlinear stretching sheet for two different types of thermal boundary conditions on the sheet, namely, constant surface temperature (CST) and prescribed surface temperature (PST). Ganji et al. [6] reported the analytical solution of the magnetohydrodynamic flow over a nonlinearly stretching sheet. Similar problems have been studied recently by Ishak et al. [7], Prasad et al. [8], Van Gorder et al. [2],
Raftari et al. [9], Abbas and Hayat [10], and Abel et al. [11], among others.

The Navier-Stokes equations alone do not adequately describe the flow properties for fluids with diversity in the physical structure which occur in most non-Newtonian fluids. Hence, the theory of micropolar fluid which was proposed by Eringen $[12,13]$ perhaps gives an accurate model for fluids where the polymeric and rotating particles need to be taken into account, by including the microrotational momentum equation in addition to the classical momentum equation. Studies of micropolar fluids have recently received considerable attention due to their applications in a number of processes that occur in industry. Such applications include suspension solutions, solidification of liquid crystals, animal bloods, and exotic lubricants. Some of the researchers who investigated such fluid with nonlinear stretching sheet are Hayat et al. [14, 15] and Rahman et al. [16], who investigated the micropolar fluid flow past a nonlinear stretching sheet taking into account the effects of a temperature-dependent viscosity and variable surface temperature. El-Aziz [17] examined the micropolar boundary-layer flow and heat transfer 
characteristics associated with a heated exponential stretching continuous sheet being cooled by a mixed convection flow, and Hsiao [18] has done an analysis for the heat and mass transfer with radiation effect of a micropolar fluid past a nonlinearly stretching sheet. Ishak et al. [19] studied the behavior of micropolar fluid flow over a fixed or continuous moving surface. The flow over a stretching/shrinking sheet immersed in a micropolar fluid has been considered by Ishak et al. [20], Yacob and Ishak [21, 22], and Rosali et al. [23], among others. They showed the existence of dual solutions for the shrinking case.

Motivated by the above investigations, the present paper studies the problem of micropolar fluid flow and heat transfer over a nonlinearly stretching plate with viscous dissipation. It is worth mentioning that viscous dissipation plays an important role in the polymer processing where it is considered like an energy source for producing heat which in turn delays the process of solidification and as a result more time is required to cool the final product (Hassan et al. [24]). It will affect the heat transfer problem as the source term does appear in the energy equation when the viscosity of the fluid or the velocity gradient is high.

\section{Problem Formulation}

Consider a steady two-dimensional laminar boundary layer flow over a nonlinearly stretching plate immersed in an incompressible micropolar fluid of ambient temperature $T_{\infty}$ as shown in Figure 1. It is assumed that the plate is stretched with velocity $U_{w}=a x^{n}$, and the plate temperature varies like $T_{w}=T_{\infty}+b x^{2 n}$, where $x$ is the distance from the slot where the plate is issued and $a, b$, and $n$ are constants. The boundary layer equations are $[25,26]$

$$
\begin{gathered}
\frac{\partial u}{\partial x}+\frac{\partial v}{\partial y}=0 \\
u \frac{\partial u}{\partial x}+v \frac{\partial u}{\partial y}=\frac{\mu+\kappa}{\rho} \frac{\partial^{2} u}{\partial y^{2}}+\frac{\kappa}{\rho} \frac{\partial N}{\partial y} \\
\rho j\left(u \frac{\partial N}{\partial x}+v \frac{\partial N}{\partial y}\right)=\gamma \frac{\partial^{2} N}{\partial y^{2}}-\kappa\left(2 N+\frac{\partial u}{\partial y}\right), \\
u \frac{\partial T}{\partial x}+v \frac{\partial T}{\partial y}=\frac{k}{\rho c_{p}} \frac{\partial^{2} T}{\partial y^{2}}+\frac{\mu+\kappa}{\rho c_{p}}\left(\frac{\partial u}{\partial y}\right)^{2},
\end{gathered}
$$

where $u$ and $v$ are the velocity components in the $x$ and $y$ directions, respectively. Further more, $\mu$ is the dynamic viscosity, $\kappa$ is the vortex viscosity (or the microrotation viscosity), $\rho$ is the fluid density, $k$ is the thermal conductivity, $c_{p}$ is the specific heat at constant pressure, $T$ is the fluid temperature, $j$ is the microinertia density, $N$ is the microrotation

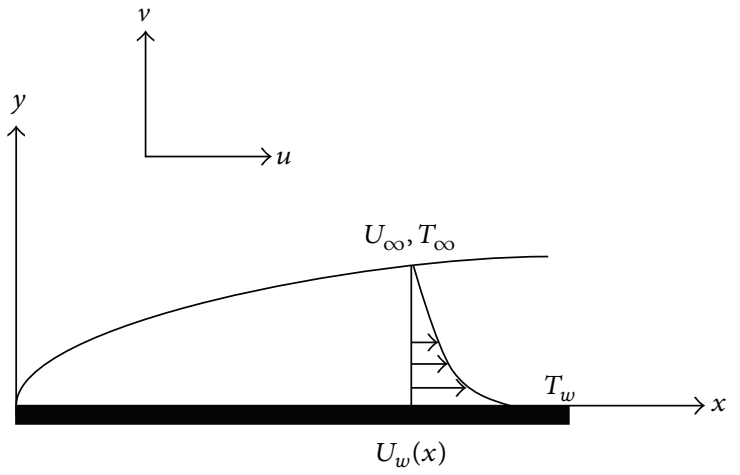

Figure 1: Physical model and coordinate system.

(or angular velocity), and $\gamma$ is the spin gradient viscosity. The boundary conditions are

$$
\begin{gathered}
u=U_{w}, \quad v=0, \quad N=-m \frac{\partial u}{\partial y}, \\
T=T_{w} \quad \text { at } y=0, \\
u \longrightarrow 0, \quad N \longrightarrow 0, \quad T \longrightarrow T_{\infty} \text { as } y \longrightarrow \infty,
\end{gathered}
$$

where $m$ is a constant with $0 \leq m \leq 1$. The case $m=1 / 2$ indicates the vanishing of antisymmetric part of the stress tensor and denotes weak concentrations (Ahmadi [27]), which is considered in the present paper. Furthermore, we follow the work of many recent authors by assuming that $\gamma=$ $(\mu+\kappa / 2) j=\mu(1+K / 2) j$, where $K=\kappa / \mu$ is the micropolar or material parameter. This assumption is invoked to allow the field of equations to predict the correct behavior in the limiting case when the microstructure effects become negligible and the total spin $N$ reduces to the angular velocity (see Ahmadi [27] or Yücel [28]).

In order to solve (1)-(4) subject to the boundary conditions (5), we introduce the following similarity transformation (see Ziabakhsh et al. [26] and Ishak et al. [29]):

$$
\begin{gathered}
\eta=\left(\frac{U_{w}}{\nu x}\right)^{1 / 2} y, \quad \psi=\left(\nu x U_{w}\right)^{1 / 2} f(\eta), \\
N=U_{w}\left(\frac{U_{w}}{v x}\right)^{1 / 2} h(\eta), \\
h(\eta)=-\frac{1}{2} f^{\prime \prime}(\eta), \quad \theta(\eta)=\frac{T-T_{\infty}}{T_{w}-T_{\infty}},
\end{gathered}
$$

where $\eta$ is the similarity variable, primes denote differentiation with respect to $\eta, v=\mu / \rho$ is the kinematic viscosity, $f$ is the dimensionless stream function, $h$ is the dimensionless microrotation, and $\psi$ is the stream function defined as $u=$ $\partial \psi / \partial y$ and $v=-\partial \psi / \partial x$ which identically satisfies (1). Using transformation (6) and utilising the boundary condition $N=$ $-(1 / 2)(\partial u / \partial y)$ from (5), (2) and (3) reduce to the single equation

$$
\left(1+\frac{K}{2}\right) f^{\prime \prime \prime}+\frac{n+1}{2} f f^{\prime \prime}-n f^{\prime 2}=0
$$


TABLE 1: Values of $\theta^{\prime}(0)$ for various values of $\operatorname{Pr}$ when $K=0, n=1$ and $\mathrm{Ec}=0$.

\begin{tabular}{lcc}
\hline $\operatorname{Pr}$ & Grubka and Bobba [31] & Present results \\
\hline 0.72 & -1.0885 & -1.088524 \\
1.0 & -1.3333 & -1.333333 \\
3.0 & -2.5097 & -2.509725 \\
10.0 & -4.7969 & -4.796873 \\
\hline
\end{tabular}

and the energy equation (4) becomes

$$
\frac{1}{\operatorname{Pr}} \theta^{\prime \prime}+\frac{n+1}{2} f \theta^{\prime}-2 n f^{\prime} \theta+\operatorname{Ec}(1+K) f^{\prime \prime 2}=0
$$

where Pr is the Prandtl number and Ec is the Eckert number defined as

$$
\operatorname{Pr}=\frac{\mu c_{p}}{k}, \quad \mathrm{Ec}=\frac{U_{w}^{2}}{c_{p}\left(T_{w}-T_{\infty}\right)} .
$$

The transformed boundary conditions are

$$
\begin{aligned}
& f(0)=0, \quad f^{\prime}(0)=1, \quad \theta(0)=1, \\
& f^{\prime}(\eta) \longrightarrow 0, \quad \theta(\eta) \longrightarrow 0 \quad \text { as } \eta \longrightarrow \infty .
\end{aligned}
$$

The physical quantities of interest are the skin friction coefficient $C_{f}$ and the local Nusselt number $\mathrm{Nu}_{x}$ which are defined as

$$
C_{f}=\frac{\tau_{w}}{\rho U_{w}^{2}}, \quad \mathrm{Nu}_{x}=\frac{x q_{w}}{k\left(T_{w}-T_{\infty}\right)},
$$

where the surface shear stress $\tau_{w}$ and the surface heat flux $q_{w}$ are given by

$$
\tau_{w}=\left[(\mu+\kappa) \frac{\partial u}{\partial y}+\kappa N\right]_{y=0}, \quad q_{w}=-k\left(\frac{\partial T}{\partial y}\right)_{y=0} .
$$

Using the dimensionless variables in (6), we obtain

$$
C_{f} \operatorname{Re}_{x}^{1 / 2}=\left(1+\frac{K}{2}\right) f^{\prime \prime}(0), \quad \frac{\mathrm{Nu}_{x}}{\operatorname{Re}_{x}^{1 / 2}}=-\theta^{\prime}(0),
$$

where $\operatorname{Re}_{x}=U_{w} x / \nu$ is the local Reynolds number.

\section{Results and Discussion}

The transformed system of (7) and (8) with the boundary conditions (10) was solved numerically using the Keller-box method as described in [30] for some values of parameters. In order to validate the numerical results obtained, we compare our results with those obtained by Grubka and Bobba [31] as shown in Table 1, which shows a favorable agreement.

Figures 2 and 3 show the effect of the nonlinear stretching parameter $n$ on the skin friction coefficient $C_{f} \operatorname{Re}_{x}^{1 / 2}$ and the local Nusselt number $\mathrm{Nu}_{x} / \mathrm{Re}_{x}^{1 / 2}$, respectively, as the material parameter $K$ varies when $\operatorname{Pr}=0.7$ and $\mathrm{Ec}=1$. As discussed by Alam et al. [32], $\operatorname{Pr}=0.7$ corresponds to air that may be

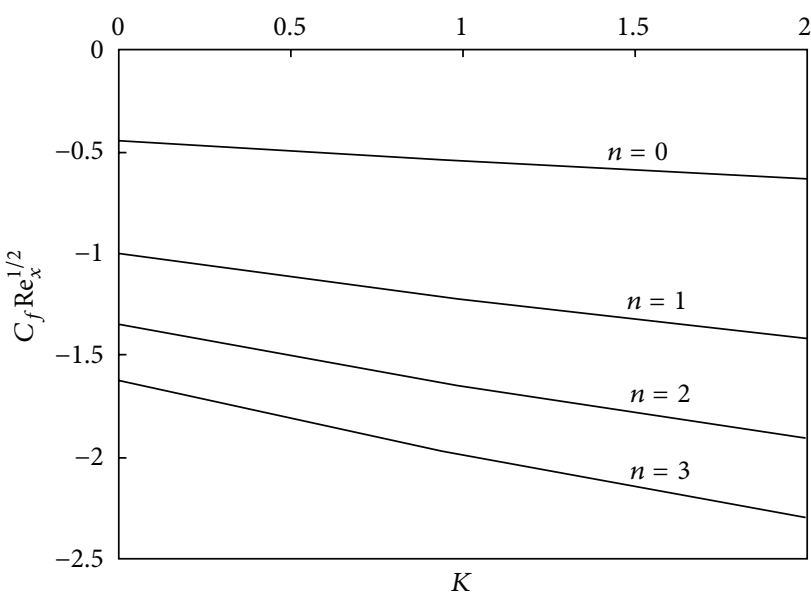

FIGURE 2: Variation of the skin friction coefficient $C_{f} \operatorname{Re}_{x}^{1 / 2}$ with $K$ for various values of $n$.

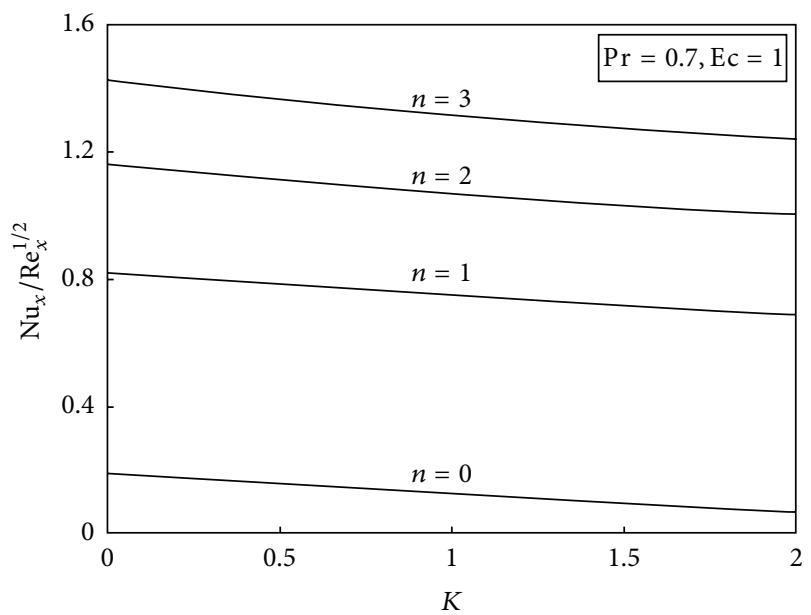

FIGURE 3: Variation of the local Nusselt number $\mathrm{Nu}_{x} / \mathrm{Re}_{x}^{1 / 2}$ with $K$ for various values of $n$ when $\operatorname{Pr}=0.7$ and $\mathrm{Ec}=1$.

considered as a micropolar fluid when it is heavily contaminated with suspended particles of dirt. It is found that all the values of the skin friction coefficients $C_{f} \mathrm{Re}_{x}^{1 / 2}$ are negative and decrease slowly as $K$ increases from $K=0$ (Newtonian fluid) to $K=2$ (micropolar fluid) for all values of the nonlinearity parameter $n$. The same trend is observed as the plate is being stretched $(n>0)$; that is, the values of the skin friction coefficient $C_{f} \operatorname{Re}_{x}^{1 / 2}$ decrease with respect to $K$. It is worth mentioning that the plate is being as more stretched, it will decrease the values of the skin friction coefficients. But opposite trend is observed for the local Nusselt number as depicted in Figure 3. As the plate is being stretched, the values of the local Nusselt number $\mathrm{Nu}_{x} / \mathrm{Re}_{x}^{1 / 2}$ are greater and for a particular value of $n$, the local Nusselt number decreases slowly as $K$ increases from 0 (Newtonian fluid) to positive values of $K$ (micropolar fluid).

The velocity and temperature distributions obtained by the finite-difference method for various values of the governing parameters are displayed in Figures 4 to 7 . Figures 4 and 5 


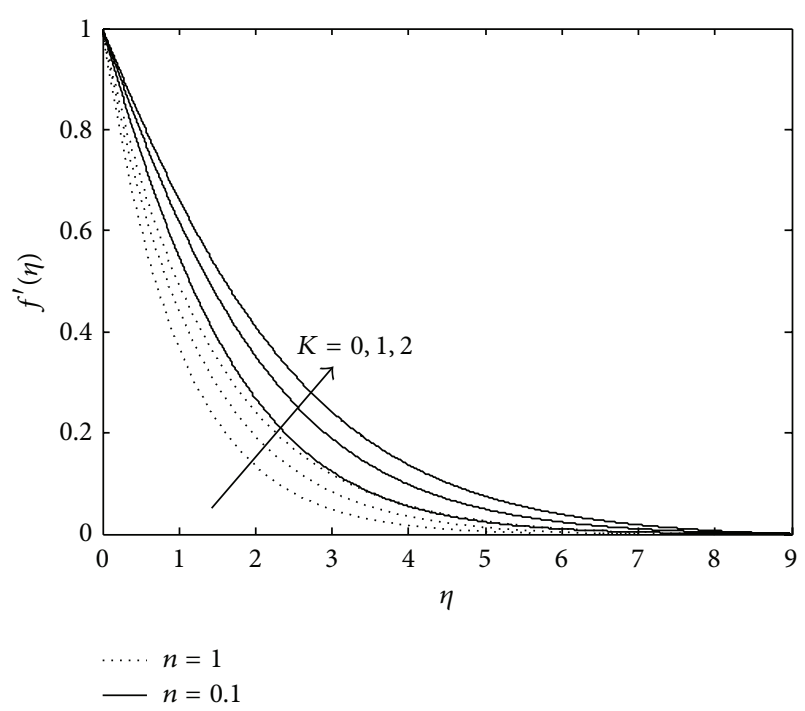

FIgURE 4: Velocity profiles $f^{\prime}(\eta)$ for various values of $K$ when $n=$ 0.1 and $n=1.0$.

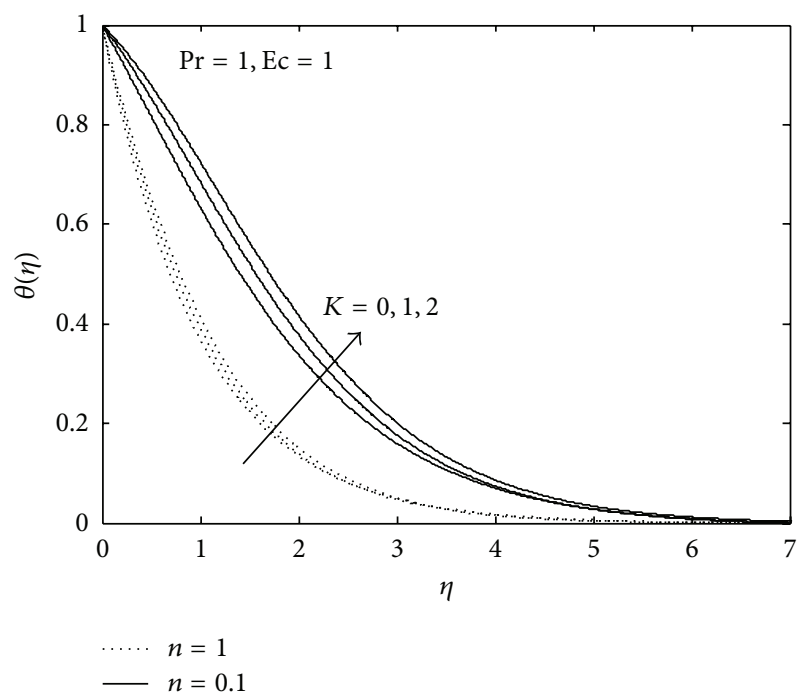

FIgURE 5: Temperature profiles $\theta(\eta)$ for various values of $K$ when $\operatorname{Pr}=1, \mathrm{Ec}=1$, and $n=0.1$ and $n=1$.

depict the effect of the micropolar/material parameter $K$ when $\operatorname{Pr}=1, \mathrm{Ec}=1$, and $n=0.1$ and $n=1.0$ on the velocity and temperature profiles. On the other hand, Figures 6 and 7 display the velocity and temperature distributions for different values of the Eckert number $\mathrm{Ec}$ when $\operatorname{Pr}=0.7, K=1$, and $n=0$ and $n=1$. It is seen that an increase in the Eckert number Ec increases the thermal boundary layer thickness, while stretching the plate decreases the thermal boundary layer. There are no changes in the velocity boundary layer thickness as illustrated in Figure 6. This is expected as the viscous dissipation only affects the thermal field. We can see that the velocity and the temperature profiles for all the figures subside monotonously to zero as $\eta$ increases which validate the boundary conditions (10), asymptotically.

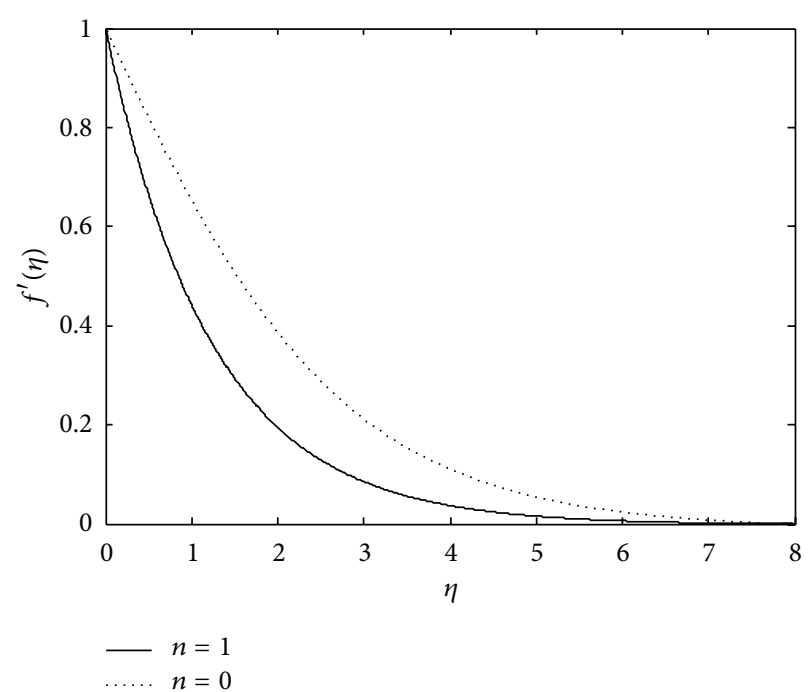

FIGURE 6: Velocity profiles $f^{\prime}(\eta)$ for $n=0$ and $n=1$.

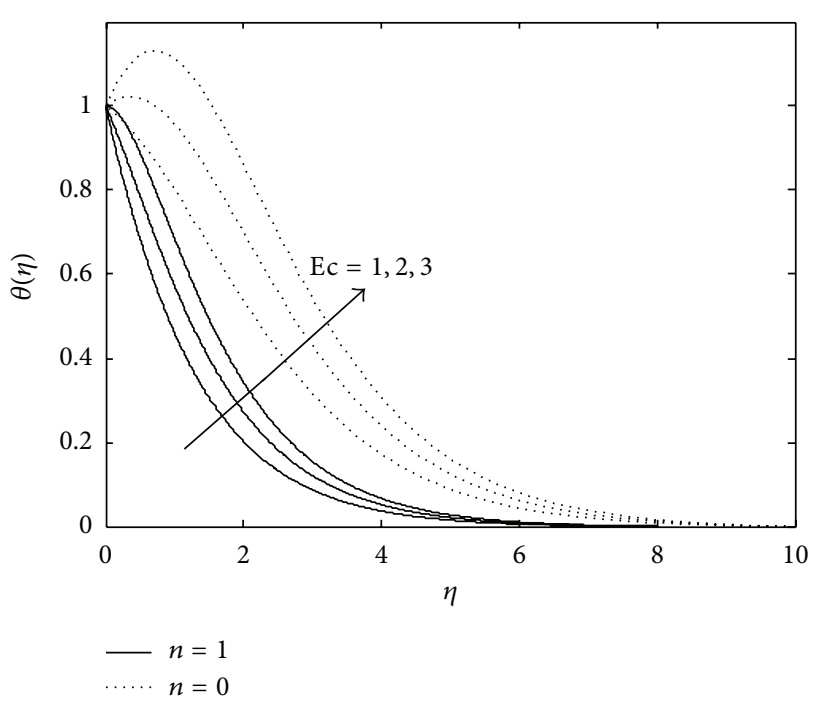

FIgURE 7: Temperature profiles $\theta(\eta)$ for various values of Ec when $\operatorname{Pr}=0.7, K=1$, and $n=0$ and $n=1$.

\section{Conclusions}

We have done a numerical investigation on the effects of the material parameter $K$, the viscous dissipation Ec, the nonlinear stretching parameter $n$, and the Prandtl number Pr on the fluid flow and heat transfer characteristics toward a nonlinear stretching sheet immersed in a micropolar fluid. It is found that both the magnitude of the skin friction coefficient and the local Nusselt number increase with the nonlinear stretching parameter $n$ and decrease when $\mathrm{K}$ increases for fixed values of $n$.

\section{Acknowledgments}

The authors wish to express their thanks to the anonymous referees for their valuable comments and suggestions. 
The financial support received from the Universiti Kebangsaan Malaysia (Project code: DIP-2012-31) is gratefully acknowledged.

\section{References}

[1] L. J. Crane, "Flow past a stretching plate," Zeitschrift für angewandte Mathematik und Physik, vol. 21, no. 4, pp. 645-647, 1970.

[2] R. A. Van Gorder, K. Vajravelu, and F. T. Akyildiz, "Existence and uniqueness results for a nonlinear differential equation arising in viscous flow over a nonlinearly stretching sheet," Applied Mathematics Letters, vol. 24, no. 2, pp. 238-242, 2011.

[3] K. Vajravelu, "Viscous flow over a nonlinearly stretching sheet," Applied Mathematics and Computation, vol. 124, no. 3, pp. 281288, 2001.

[4] R. Cortell, "Viscous flow and heat transfer over a nonlinearly stretching sheet," Applied Mathematics and Computation, vol. 184, no. 2, pp. 864-873, 2007.

[5] R. Cortell, "Heat and fluid flow due to non-linearly stretching surfaces," Applied Mathematics and Computation, vol. 217, no. 19, pp. 7564-7572, 2011.

[6] D. D. Ganji, H. Bararnia, S. Soleimani, and E. Ghasemi, "A nalytical solution of the magneto-hydrodynamic flow over a nonlinear stretching sheet," Modern Physics Letters B, vol. 23, no. 20-21, pp. 2541-2556, 2009.

[7] A. Ishak, R. Nazar, and I. Pop, "Unsteady mixed convection boundary layer flow due to a stretching vertical surface," The Arabian Journal for Science and Engineering B, vol. 31, no. 2, pp. 165-182, 2006.

[8] K. V. Prasad, K. Vajravelu, and P. S. Datti, "Mixed convection heat transfer over a non-linear stretching surface with variable fluid properties," International Journal of Non-Linear Mechanics, vol. 45, no. 3, pp. 320-330, 2010.

[9] B. Raftari, S. T. Mohyud-Din, and A. Yildirim, "Solution to the MHD flow over a non-linear stretching sheet by homotopy perturbation method," Science China, vol. 54, no. 2, pp. 342-345, 2011.

[10] Z. Abbas and T. Hayat, "Stagnation slip flow and heat transfer over a nonlinear stretching sheet," Numerical Methods for Partial Differential Equations, vol. 27, no. 2, pp. 302-314, 2011.

[11] M. S. Abel, K. A. Kumar, and R. Ravikumar, "MHD flow and heat transfer with effects of buoyancy, viscous and Joules dissipation over a nonlinear vertical stretching porous sheet with partial slip," Engineering, vol. 3, pp. 285-291, 2011.

[12] A. C. Eringen, “Theory of micropolar fluids," vol. 16, pp. 1-18, 1966.

[13] A. C. Eringen, "Theory of thermomicrofluids," Journal of Mathematical Analysis and Applications, vol. 38, no. 2, pp. 480496, 1972.

[14] T. Hayat, Z. Abbas, and T. Javed, "Mixed convection flow of a micropolar fluid over a non-linearly stretching sheet," Physics Letters A, vol. 372, no. 5, pp. 637-647, 2008.

[15] T. Hayat, T. Javed, and Z. Abbas, "MHD flow of a micropolar fluid near a stagnation-point towards a non-linear stretching surface," Nonlinear Analysis. Real World Applications, vol. 10, no. 3, pp. 1514-1526, 2009.

[16] M. M. Rahman, M. A. Rahman, M. A. Samad, and M. S. Alam, "Heat transfer in a micropolar fluid along a non-linear stretching sheet with a temperature-dependent viscosity and variable surface temperature," International Journal of Thermophysics, vol. 30, no. 5, pp. 1649-1670, 2009.

[17] M. A. El-Aziz, "Viscous dissipation effect on mixed convection flow of a micropolar fluid over an exponentially stretching sheet," Canadian Journal of Physics, vol. 87, no. 4, pp. 359-368, 2009.

[18] K. L. Hsiao, "Heat and mass transfer for micropolar flow with radiation effect past a nonlinearly stretching sheet," Heat and Mass Transfer, vol. 46, no. 4, pp. 413-419, 2010.

[19] A. Ishak, R. Nazar, and I. Pop, "Boundary-layer flow of a micropolar fluid on a continuous moving or fixed surface," Canadian Journal of Physics, vol. 84, no. 5, pp. 399-410, 2006.

[20] A. Ishak, Y. Y. Lok, and I. Pop, "Stagnation-point flow over a shrinking sheet in a micropolar fluid," Chemical Engineering Communications, vol. 197, no. 11, pp. 1417-1427, 2010.

[21] N. A. Yacob and A. Ishak, "Micropolar fluid flow over a shrinking sheet," Meccanica, vol. 47, no. 2, pp. 293-299, 2012.

[22] N. A. Yacob and A. Ishak, "Stagnation point flow towards a stretching/shrinking sheet in a micropolar fluid with a convective surface boundary condition," Canadian Journal of Chemical Engineering, vol. 90, no. 3, pp. 621-626, 2012.

[23] H. Rosali, A. Ishak, and I. Pop, "Micropolar fluid flow towards a stretching/shrinking sheet in a porous medium with suction," International Communications in Heat and Mass Transfer, vol. 39, pp. 826-829, 2012.

[24] H. Hassan, N. Regnier, C. Pujos, and G. Defaye, "The effect of viscous dissipation on the polymer temperature during injection molding," in Proceedings of the 5th European ThermalSciences Conference, Eindhoven, The Netherlands, 2008.

[25] A. Ishak, R. Nazar, and I. Pop, "Post-stagnation-point boundary layer flow and mixed convection heat transfer over a vertical, linearly stretching sheet," Archives of Mechanics, vol. 60, no. 4, pp. 303-322, 2008.

[26] Z. Ziabakhsh, G. Domairry, and H. Bararnia, "Analytical solution of non-Newtonian micropolar fluid flow with uniform suction/blowing and heat generation," Journal of the Taiwan Institute of Chemical Engineers, vol. 40, no. 4, pp. 443-451, 2009.

[27] G. Ahmadi, "Self-similar solution of imcompressible micropolar boundary layer flow over a semi-infinite plate," International Journal of Engineering Science, vol. 14, no. 7, pp. 639-646, 1976.

[28] A. Yücel, "Mixed convection in micropolar fluid flow over a horizontal plate with surface mass transfer," International Journal of Engineering Science, vol. 27, no. 12, pp. 1593-1602, 1989.

[29] A. Ishak, R. Nazar, and I. Pop, "Heat transfer over a stretching surface with variable heat flux in micropolar fluids," Physics Letters A, vol. 372, no. 5, pp. 559-561, 2008.

[30] T. Cebeci and P. Bradshaw, Physical and Computational Aspects of Convective Heat Transfer, Springer Study Editions, Springer, New York, NY, USA, 1988.

[31] L. J. Grubka and K. M. Bobba, "Heat transfer characteristics of a continuous, stretching surface with variable temperature," Journal of Heat Transfer, vol. 107, no. 1, pp. 248-250, 1985.

[32] M. S. Alam, M. A. Sattar, M. M. Rahman, and A. Postelnicu, "Local similarity solution of unsteady hydromagnetic convection flow of a micropolar fluid along a continuously moving permeable plate," International Journal of Heat and Technology, vol. 28, no. 2, pp. 95-105, 2010. 


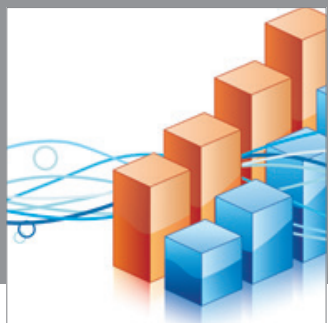

Advances in

Operations Research

mansans

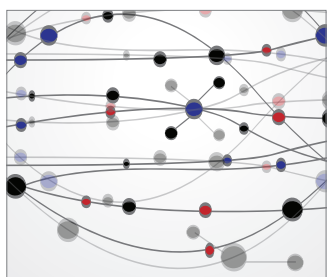

The Scientific World Journal
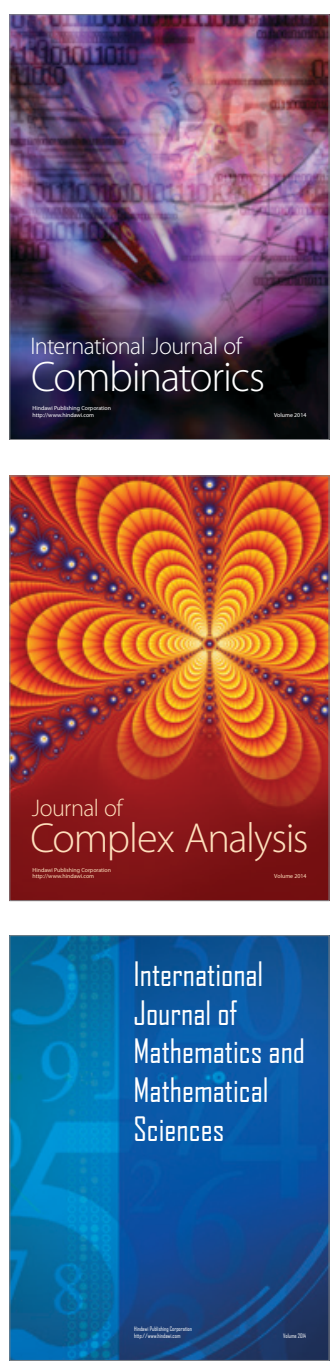
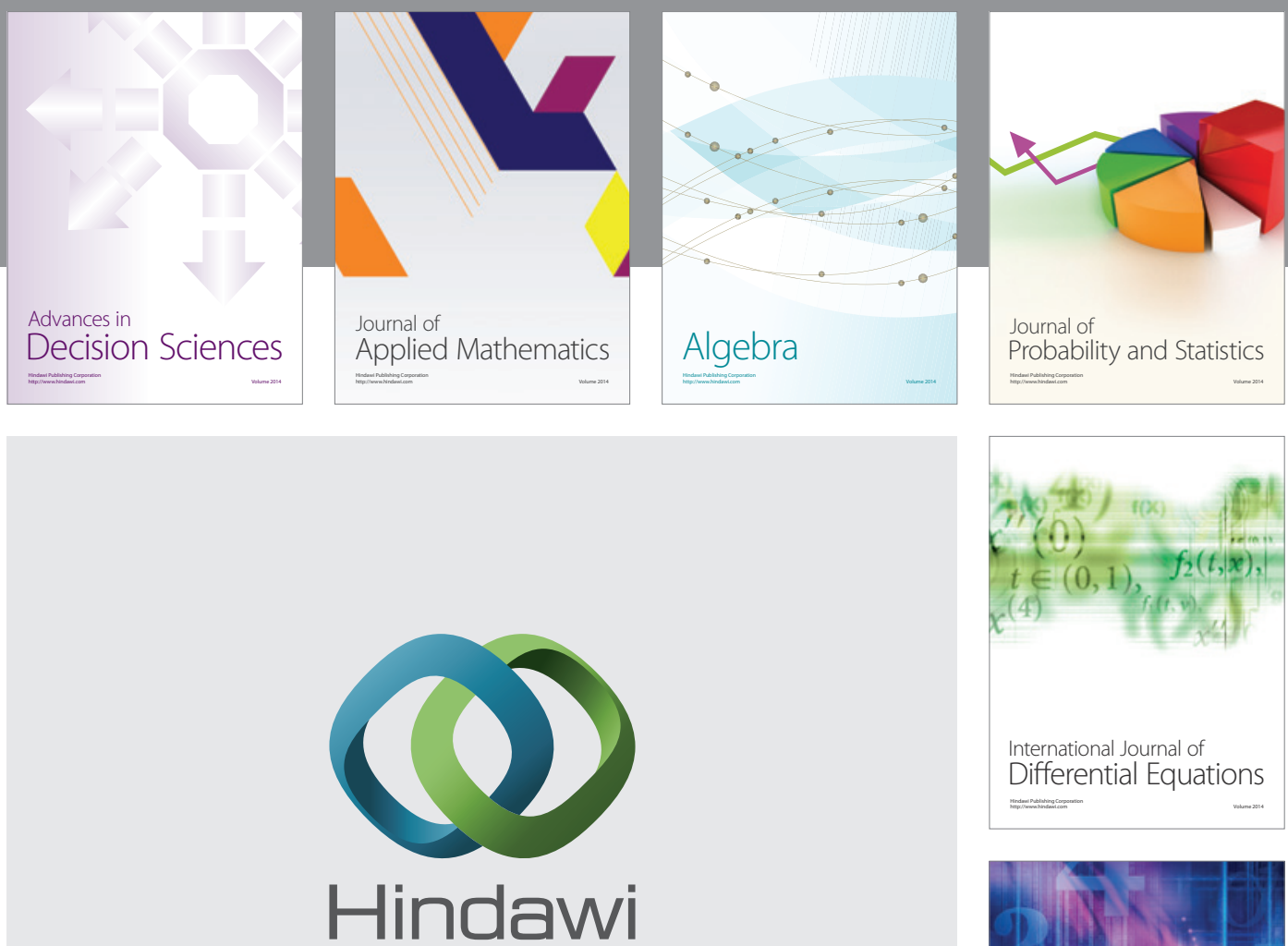

Submit your manuscripts at http://www.hindawi.com
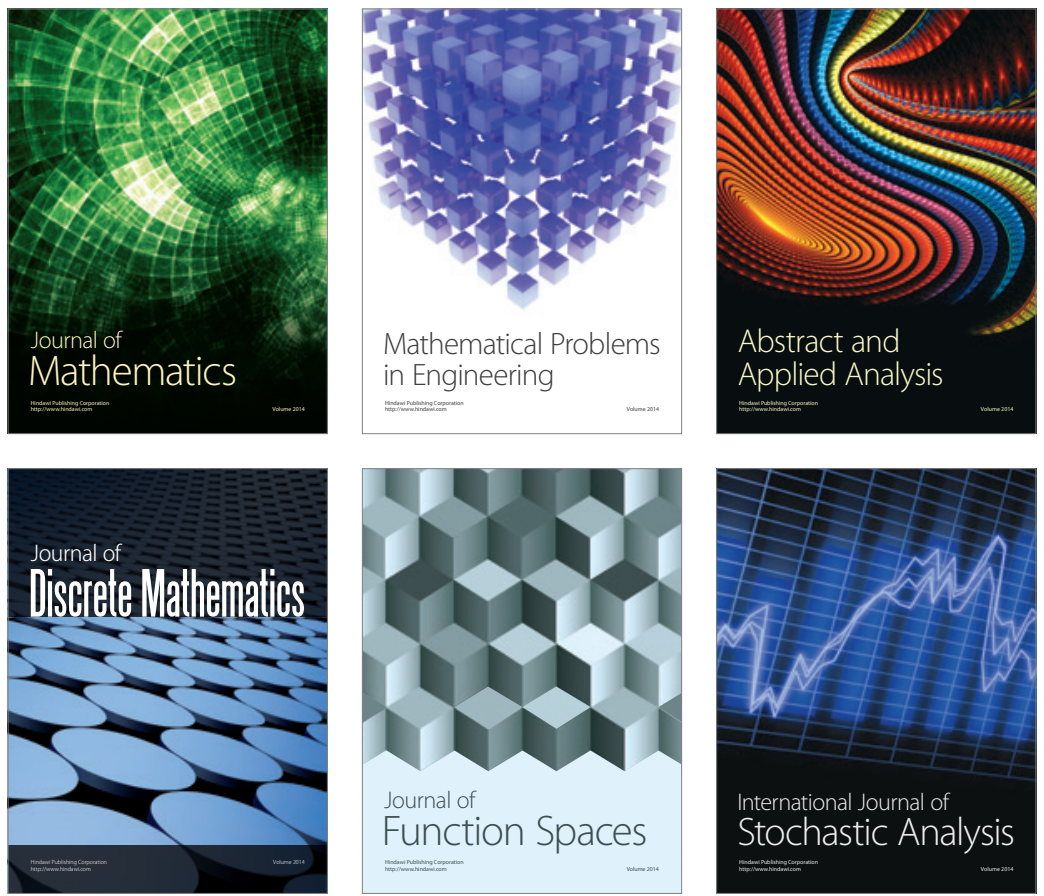

Journal of

Function Spaces

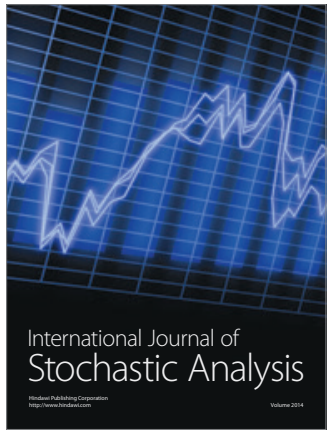

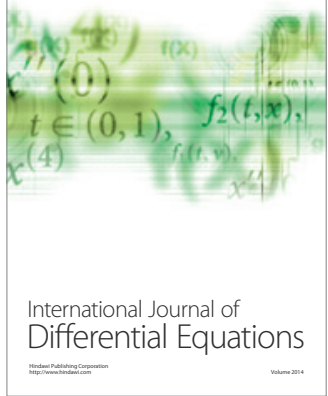
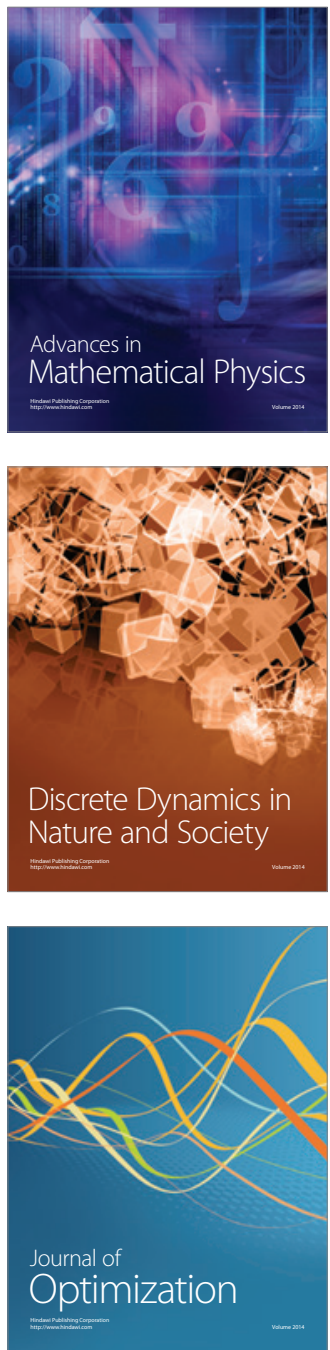